\title{
Prey dispersal and predator persistence
}

\author{
V.A.A. Jansen ${ }^{\mathrm{a}}$ and M.W. Sabelis ${ }^{\mathrm{b}}$ \\ ${ }^{a}$ Institute of Theoretical Biology, University of Leiden, Leiden, Netherlands \\ ${ }^{b}$ Department of Pure and Applied Ecology, University of Amsterdam, Amsterdam, Netherlands
}

\begin{abstract}
Jansen, V.A.A. and Sabelis, M.W., 1992. Prey dispersal and predator persistence. Exp. Appl. Acarol,, 14: $215-231$.

To understand how patchiness influences population dynamics of a tri-trophic interaction, a tractable model is formulated in terms of differential equations. Motivated by the structure of systems such as plants, phytophagous mites and predatory mites, the model takes dispersal into account at the middle trophic level. The effect of dispersal for the middle level in a tri-trophic system could be either stabilising or destabilising since the middle level acts both as prey and as predator. First a simple model with logistic growth for the lowest level is formulated. A model with logistic growth for the lowest level and instantaneous dispersal has a globally stable three-species equilibrium, if this equilibrium exists. Addition of a middle level dispersal phase of non-negligible duration influences equilibrium stability. In the absence of the top trophic level a limit cycle can occur, caused by the delay that exists in the reaction of the middle level to the changes in the lowest level. With low predator efficiency, it is also possible to have an unstable three-species equilibrium. So addition of a middle level dispersal phase of non-negligible duration can work destabilising. Next the persistence of the third trophic level is studied. Even when the three-species equilibrium exists, the third trophic level need not be persistent. A two-species limit cycle can keep its stability when a three-species equilibrium exists; the system is then bistable. It is argued that such a bistability can offer an alternative explanation for pesticide-induced outbreaks of spider mites and failure of predator introduction.
\end{abstract}

\section{INTRODUCTION}

Spatial heterogeneity is notoriously difficult to model. Often the only possibility is to model all individuals separately and one is subsequently confronted with the formidable task of drawing clear conclusions from complicated simulation studies. In most analytical models spatial heterogeneity is ignored. To a certain extent these models can be expected to hold for nonuniformly distributed populations. If, however, individuals respond to the local population density rather than to overall density, we can expect an influence of the spatial distribution on population dynamics. By formulating ana-

Correspondence to: V.A.A. Jansen, Institute of Theoretical Biology, University of Leiden, P.O. Box 9516, 2300 RA Leiden, Netherlands. 
lytically tractable models that describe an extreme case of spatial heterogeneity we attempt to bridge some of the gap that exists between these two approaches.

When local populations are connected through occasional dispersal events only, we have a situation in which individuals only respond to local conditions. Suppose local populations have a certain probability of going extinct and new ones are formed by colonisation of suitable habitat from still existing populations. The sort of dynamics that the ensemble of local populations has, can be very different from the behaviour of one big population. If all individuals belong to a single population, persistence would not be possible because sooner or later the population would go extinct whereas the collection of local populations can persist (Reddingius and Den Boer, 1970; Levin, 1976; Zeigler, 1977; Crowley, 1978, 1981; Verboom et al., 1991).

A population consisting of a collection of local populations as described above is normally referred to as a metapopulation. The concept of a metapopulation is used to illustrate the fact that, although populations on a local scale go extinct, persistence on a regional scale still can be possible. Local populations can exist because only islands or patches in total space are suitable for a species, as is naturally the case with host-inhabiting species such as plant inhabitants, pathogens or parasitoids. The work in this paper is largely motivated by a system of plant-inhabiting mites and predatory mites. These mites do tend to occur in patches, and the concept of a metapopulation can be used to describe their population dynamics.

The consequence of living in a patchy environment is that two different modes of movement exist: movement within patches and movement between patches. If mean successful movement between patches is of non-negligible duration it will have an effect on population dynamics. Since dispersal between patches is inseparable from a patchy environment we will investigate what effect a dispersal phase of non-negligible duration has on stability. Thus we are able to capture an important feature of spatial heterogeneity in a tractable model.

A dispersal phase can have a stabilising effect when it concerns prey, and a destabilising one when it concerns predators (Diekmann et al., 1988; Sabelis et al., 1991). When plant dynamics is taken into account, the effect of predator dispersal will not change dramatically. The situation is different in the case of dispersing prey. Herbivore prey have a double role: they are prey to their predators, but are predators for the plants, and the respective stabilising and destabilising effects will interact. In this paper we will investigate the effect of a herbivore dispersal phase on stability in a tri-trophic system.

In natural ecosystems spider mites are normally present in low densities. The widespread intensive use of organic pesticides after world war II induced outbreaks of spider mites. The explanation frequently given for this is that after spraying, both prey and predator are suppressed. For the surviving prey, 
life now is good (less predators); for the surviving predators life is bad because food is harder to find. This does explain for a rise in the prey density to levels higher than before pesticide application but does not explain for total predator extinction and continued outbreaks. We will demonstrate using a simple tri-trophic model that predator extinction is possible after perturbation from a stable steady state and that predator extinction critically depends on the size of the perturbation. Moreover, we will show that success of predator introductions hinges on the number of predators released.

\section{A SIMPLE PREDATOR-PREY PATCH MODEL}

The method we will use to investigate the effect of a prey dispersal phase on model stability is the same as the touchstone technique used in Sabelis and Diekmann (1988) and Sabelis et al. (1991): first a simple model is formulated and the stability of its equilibria determined. Then some further process is added to this simple model. By comparing the stabilities of the equilibria of both models, it is concluded whether the process under study acts in a stabilising or destabilising manner. As a touchstone we will use a simple tritrophic model to which we will add herbivore dispersal.

We simplify our view to a caricature of prey-predator interaction in a patchy environment as schematically indicated in Figs. 1a and 1b. For the sake of simplicity we will call the trophic levels plant, herbivore and predator, but other interpretations are possible, of course. The prey in our model are herbivores and they can only live on plants. These plants form suitable patches in a world which is unsuitable as a rule. Some plants are inhabited by herbivores who feed and reproduce there (Fig. 1a). After some time the resources
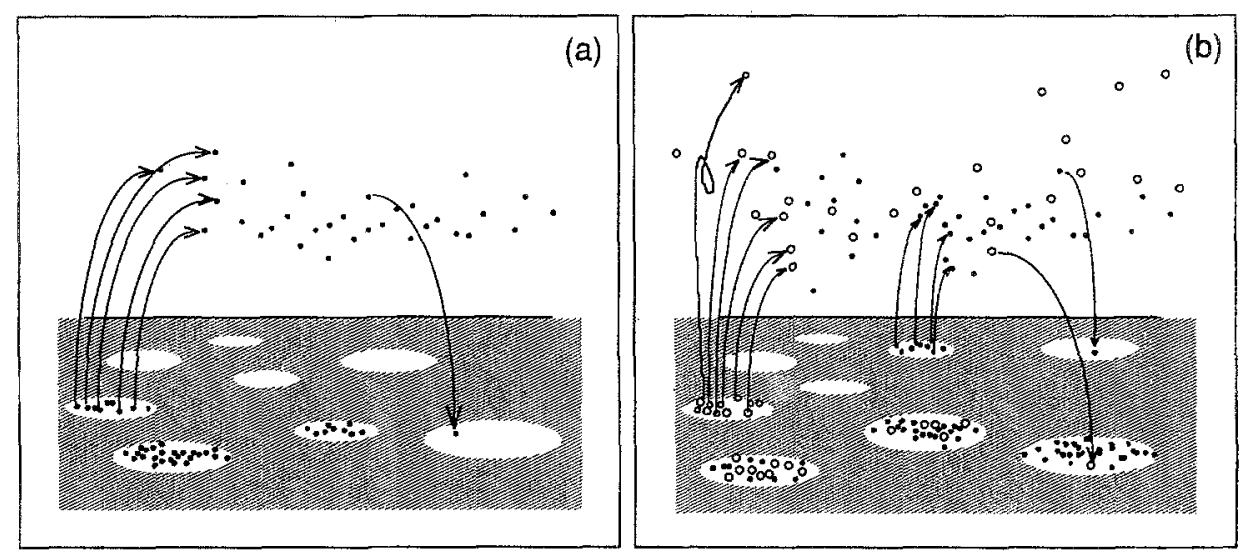

Fig. 1. (a) A simple universe with free plant patches, herbivore patches and dispersing herbivores. (b) the same universe with herbivore-predator patches and dispersing predators. Open spots: patches, $\bullet$ : herbivore, $O$ : predator. 
of a plant get exhausted because of the growing number of herbivores that feed on it. The residing herbivores then leave this patch, they disperse and search for new uninhabited patches. Most dispersing animals do not find empty patches and die. Some dispersers are lucky and find a new patch, occupy it and start reproducing there until after some time also this patch gets exhausted and the process repeats itself.

The world as conceived by the predators is one of unoccupied patches that are converted into prey patches that live for some time and then disappear, as is sketched in Fig. 1a. Predators search for these herbivore patches. Once a predator finds a herbivore patch, it stays there and starts reproducing at the expense of the herbivores present in the patch. A predator and its offspring stay in the patch until no more prey is left. Then the predators too leave the patch, disperse and search for new herbivore patches. Figure $1 \mathrm{~b}$ shows our simple world of plants, herbivores and predators.

The model used as a touchstone in Sabelis and Diekmann (1988) and Sabelis et al. (1991) is the well-known Lotka-Volterra model in which the variables are interpreted as the number of patches instead of number of individuals. The Lotka-Volterra model is neutrally stable and as a consequence of that, structurally unstable. This means that small changes in the equations can have a profound influence on the dynamics. This structural instability is often considered the major shortcoming of the Lotka-Volterra model, because it implies an extreme sensitivity to the precise nature of simplifications of reality that have been made. On the other hand, it is precisely this property of the Lotka-Volterra model that makes it an ideal instrument for the use as a touchstone.

While the Lotka-Volterra model can indeed serve as a touchstone model because its dynamical properties are on the knife's edge between stability and instability, there is no such neutrally stable touchstone for tri-trophic interactions. As an alternative we will use the following tri-trophic null model:

$$
\begin{aligned}
& \frac{\mathrm{d} R}{\mathrm{~d} t}=r R\left(1-\frac{R}{c}\right)-\zeta R N \\
& \frac{\mathrm{d} N}{\mathrm{~d} t}=\zeta R N-k N-\eta N M \\
& \frac{\mathrm{d} M}{\mathrm{~d} t}=\eta N M-\nu M
\end{aligned}
$$

The variable $R$ denotes the number of available free patches, and can be thought of as plant density. We assume that patches are 'disposable': depleted patches disappear like wilted leaves shrivel and fall. Patches that are occupied by herbivores do not inhibit growth of free patches as much as unoccupied 
patches. For simplicity growth of free patches is assumed to be independent of occupied patches. The number of free patches grows logistic, with initial growth rate $r$ and carrying capacity $c$. In the presence of herbivores free patches are discovered and converted into herbivore patches. Prey dispersers are assumed to act independently, and the duration of the dispersal phase is assumed to be negligible. The number of prey dispersers is therefore proportional to the number of prey patches that get exhausted. The number of free patches discovered per unit of time equals $\zeta R N$; this also amounts to the assumption that the probability for each free patch of being discovered is independent of the density of free patches. This assumption will be valid as long as the regions of attraction of free patches do not overlap.

A free patch that is discovered by a herbivore is by definition converted to a herbivore patch and so the rate of formation of herbivore patches equals $\zeta R N$. Herbivore patches disappear for two reasons, a patch can get exhausted or it can be discovered by predators. These patches have an exponentially distributed lifetime in absence of predators and thus a constant fraction gets exhausted per unit of time, say $k N$. Because of patch disposability these depleted patches do not come back as free patches.

The number of herbivore patches that are discovered per unit of time by predators is proportional to $M$, the number of predator patches (here, we assume that dispersing and searching does not take time for predators either). The number of discovered patches also depends on the number of herbivore patches available for predators. If all herbivore patches have a constant probability of being discovered (that is no overlapping attractive regions), the number of herbivore patches discovered per unit of time by predators is given by $\eta N M$ with $\eta$ again a constant.

The number of predator patches increases because of discovered herbivore patches; this amounts to $\eta N M$ per unit of time. It decreases because of patches getting depleted of preys. We assume that per unit of time all predator patches have an equal probability $\nu$ of getting depleted. Although patches in which all herbivores are removed, could be re-invaded by herbivores, we assume that the number of herbivores that still could be produced in such patch is negligible compared to the production of a truly free patch. Therefore the depleted predator patches are not added to the free patches.

This model has been analysed before (Freedman and Waltman, 1977) and we will recapitulate the main results. In the absence of herbivores and predators the plants grow to their carrying capacity. If the carrying capacity is chosen smaller than $k / \zeta$, the equilibrium with plants at carrying capacity and neither herbivores nor predators is stable in the presence of herbivores. This means that the herbivores cannot invade the system. With a carrying capacity chosen bigger than this threshold, the 'plants only' equilibrium becomes unstable with respect to the herbivores. If herbivores are added, invasion will 
now be successful and the densities will go to a new equilibrium with plant density below carrying capacity and positive herbivore density. This equilibrium is stable as long as predators are absent. For moderate carrying capacities $(k / \zeta<c<(k / \zeta) /(1-\zeta \nu / m \eta))$ it is also stable in presence of predators: if predators are added they will produce less than one new predator each and they will disappear again. The densities of plants and herbivores will go back to that of the two-species equilibrium.

With the carrying capacity chosen such that each introduced predator will produce at least one new predator $(c>(k / \zeta)(1-\zeta \nu / r \eta))$, invasion of predators is possible and now the equilibrium with plants and herbivores becomes unstable with respect to predators (still it is stable in the sense that in the absence of predators the densities of herbivores and plants will go to these values. ) In this case there also exists a three-species equilibrium and local stability analysis reveals that this equilibrium is always stable. It is even possible to show that this equilibrium is globally stable (Harrison, 1978; Gard and Hallam, 1979; So, 1979; Hofbauer and Sigmund, 1988), meaning that any initial conditions with positive plant, herbivore and predator density will eventually end up in this three-species equilibrium.

The equilibria of this model, are not neutrally stable. The reason why is not difficult to see: in the plant level we included logistic growth and this has a stabilising effect. A straightforward way to get rid of this stabilising effect would be to replace the logistic growth with exponential growth, as in the Lotka-Volterra model. This can easily be done by taking the limit of the carrying capacity tending to infinity. The consequence of this is, however, that also the three-species equilibrium disappears into infinity and that we end up with no equilibrium to compare stability with. Therefore we have chosen this model with a stable three-species equilibrium above the alternative with no equilibrium at all (see Sabelis et al. (1991) for the alternative choice). This choice puts some restrictions on the use of the touchstone technique. Because of the bias towards stability we will only be able to get results on destabilisation. Next we will extend this model with a herbivore dispersal phase and compare stabilities of the equilibria.

\section{HERBIVORE DISPERSAL IN A TRI-TROPHIC CONTEXT}

In the previous model it was assumed that the rate of formation of herbivore patches is proportional to the number of herbivore patches. If prey dispersal is of non-negligible duration this assumption is no longer valid. Formation of new herbivore patches then depends on the number of dispersing prey which is no longer a simple multitude of the number of herbivore patches. We will explicitly describe the number of herbivores that are dispersing with the variable $P$. Again we assume a constant probability per free patch to be 
discovered and independent dispersal and so the number of patches formed per time unit becomes $\gamma R P$.

Once a herbivore patch is founded it has, as before, an exponential life time in the absence of predators. The number of herbivore patches that go extinct per unit of time equals $k N$. A patch that goes extinct contributes $z$ dispersers to $P$, the total number of dispersing herbivores. Dispersing herbivores can disappear for all sorts of reasons but one: they cannot be found and eaten by predators. We assume a constant death rate $\mu$.so that the life time of dispersing herbivores is distributed exponentially. The dynamics of the predators is the same as in system (1). In the presence of predators a quantity $\eta N M$ of herbivore patches per unit of time is converted into predator-prey patches. This assumes independent predator dispersal, predator dispersal of non-negligible duration, no overlap in the attractive regions of the herbivore patches and a constant fraction of predator-prey patches becoming depleted of prey per unit of time. This fraction equals $\nu M$. All these considerations result in the equations:

$$
\begin{aligned}
& \frac{\mathrm{d} R}{\mathrm{~d} t}=r R\left(1-\frac{R}{c}\right)-\gamma R P \\
& \frac{\mathrm{d} N}{\mathrm{~d} t}=\gamma R P-k N-\eta N M \\
& \frac{\mathrm{d} P}{\mathrm{~d} t}=k z N-\mu P \\
& \frac{\mathrm{d} M}{\mathrm{~d} t}=\eta N M-\nu M
\end{aligned}
$$

First we observe that by taking the limit of $\mu$ and $z$ tending to infinity, keeping the ratio $\mu / z$ constant, the dispersal phase is again of nil duration and the model becomes the null model again. We will analyse the extended model in the same fashion as we analysed the tri-trophic null model, beginning with absent predators and then later consider the behaviour in the presence of predators.

\section{Dynamics with only plants and herbivores present}

In the absence of predators and herbivores plant growth is logistic and the model is identical to the tri-trophic null model. With herbivores present but predators absent we have to consider the system (2) with $M=0$. With low carrying capacity the introduction of herbivores just results in extinction of herbivores. The herbivores cannot establish themselves: the 'plants only' equilibrium is stable (with respect to herbivores) and a plant-herbivore equilibrium does not exist. For bigger carrying capacities the herbivores can establish themselves: now the plant-herbivore equilibrium also exists. For 
moderate values of the carrying capacity all is the same as in the null model, the plant-herbivore equilibrium is stable and after introduction of herbivores densities will settle down at equilibrium values.

All this changes if we increase the carrying capacity above a certain critical value. The plant-herbivore equilibrium then loses its stability to a limit cycle, which attracts all orbits. An introduction of a small amount of herbivores in a plant population that is at carrying capacity, will result in an increase in herbivore patches and a decrease in the density of free patches. Because of the lack of replenishment the herbivore density will subsequently go down. Instead of damping out, the cycle will enhance itself and finally the system will show stable oscillating behaviour. This means that a non-instantaneous dispersal phase for herbivores causes instability. What we have here is the effect of herbivore dispersal with herbivores in the role of plant predators. Indeed predator dispersal works destabilising and this is exactly what we find here.

How big exactly the threshold, above which the plant-herbivore system shows sustained cycles, is, depends on some of the model parameters concerning plants and herbivores. To show of what form this dependence is we introduce the new parameters $t$, the reciprocal of the total average herbivore patch lifetime including time needed for dispersal relative to initial plant growth and $f$, the fraction of total patch lifetime that is devoted to dispersal. The parameter $t$ is simply the harmonic mean of $\mu / r$ and $k / r$ :

$\frac{1}{t}=\frac{r}{\mu}+\frac{r}{k} \Leftrightarrow t=\frac{\mu k}{r(\mu+k)}$

and $f$ equals mean disperser lifetime divided by mean total herbivore lifetime which can be computed from

$f=\frac{1 / \mu}{1 / r t}=r \frac{t}{\mu}=\frac{k}{\mu+k}$

In Fig. 2 the stable and unstable domains of the herbivore-plant equilibrium are plotted. These domains are separated by the threshold for limit cycle behaviour. This figure shows that an increase in mean patch lifetime can bring the system from the unstable to the stable domain. If we keep mean total patch lifetime $(1 / t)$ constant we see that an increase in the relative importance of the dispersal phase $(f)$ destabilises the model as long as the dispersal phase occupies less than half of the total lifetime. This can be visualised in Fig. 2 by walking along a horizontal line from left to right. It is possible to come from the stable into the unstable domain. This is not very surprising because a predator dispersal phase is destabilising. If, however, it occupies more than half of the total lifetime we see that with a further increase in relative importance becomes stabilising again! Hence, we find maximum insta- 


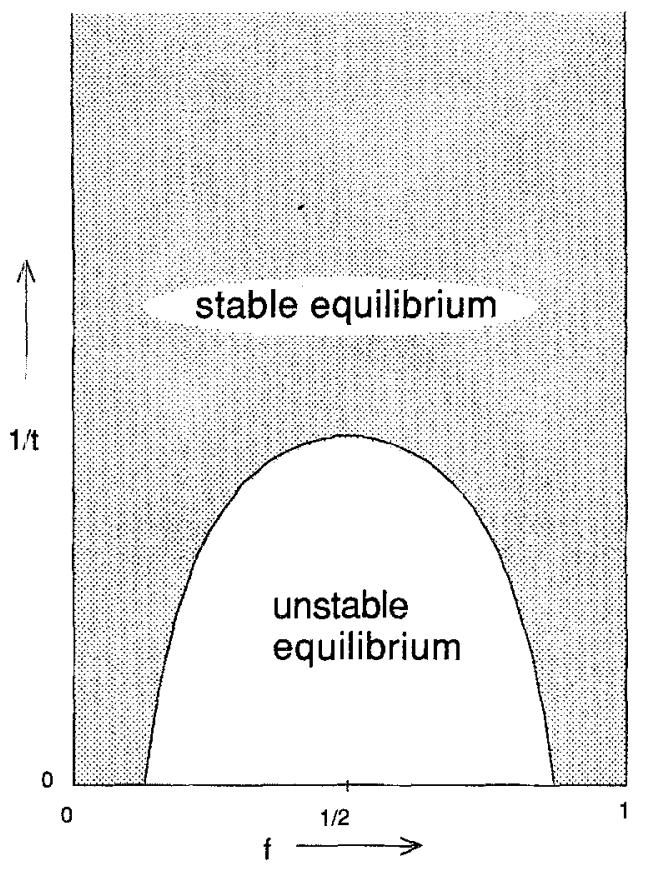

Fig. 2. Stable and unstable domains of the two-species equilibrium as related to total mean herbivore patch lifetime $(1 / t)$ and fraction of total herbivore patch lifetime devoted to dispersal $(f)$.

bility when the dispersal phase occupies exactly half of the total lifetime or, in other words when dispersal takes as much time as does consuming the patch.

In Fig. 3 the probability of forming a daughter patch is plotted against the age of a patch. The parameters $r, \mu$ and $k$ are chosen such that the mean total lifetime $1 / t$ is the same for all drawn distributions, but that the relative importance of the dispersal phase varies. In Fig. 2 this would give a collection of points that are on a horizontal line. We see that as the relative importance of the dispersal phase gets closer to $1 / 2$ there is an increasing delay in the onset of the formation of new patches. It is this delay that causes instability. The fact that this delay is maximal when the time spent as a disperser equals patch lifetime depends on the chosen exponential distributions for patch and disperser survival. The biological significance of these observations lies first of all in the fact that a delay in the formation of daughter patches can cause instability.

Now that we know why stable oscillations occur, we will turn our attention to the appearance of these cycles. Figure 4 a shows a typical sequence of cycles, Fig. $4 \mathrm{~b}$ is a blow up of one such cycle. What happens is that after a peak in disperser density the densities of herbivore patches and free patches are low. Then a build-up period follows in which the density of free patches grows 


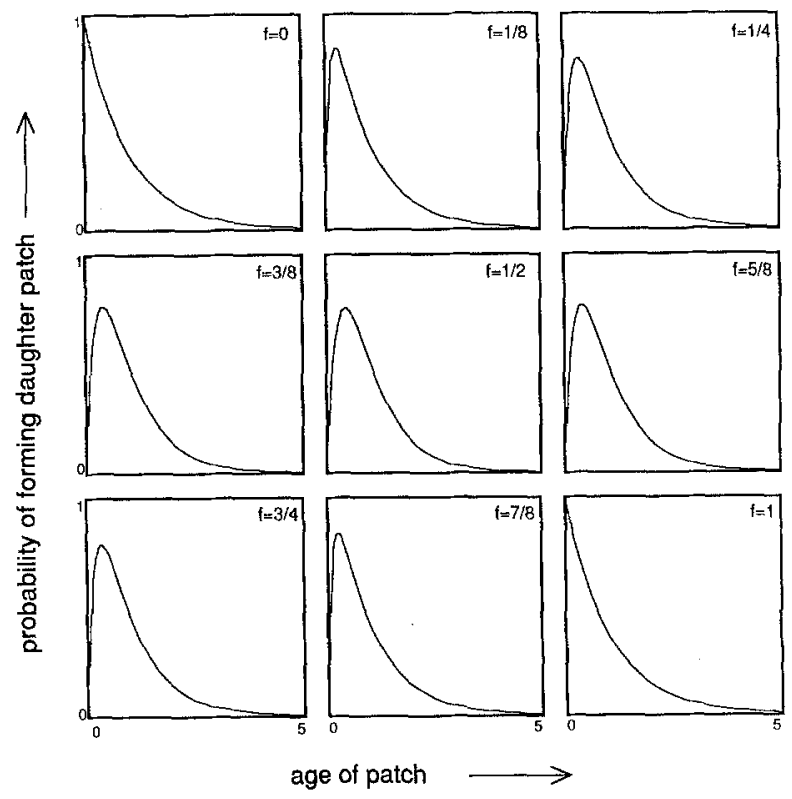

Fig. 3. The probability of forming daughter patches versus age of a patch for various values of $f$, the fraction of herbivore patch lifetime devoted to dispersal, $t=1$.
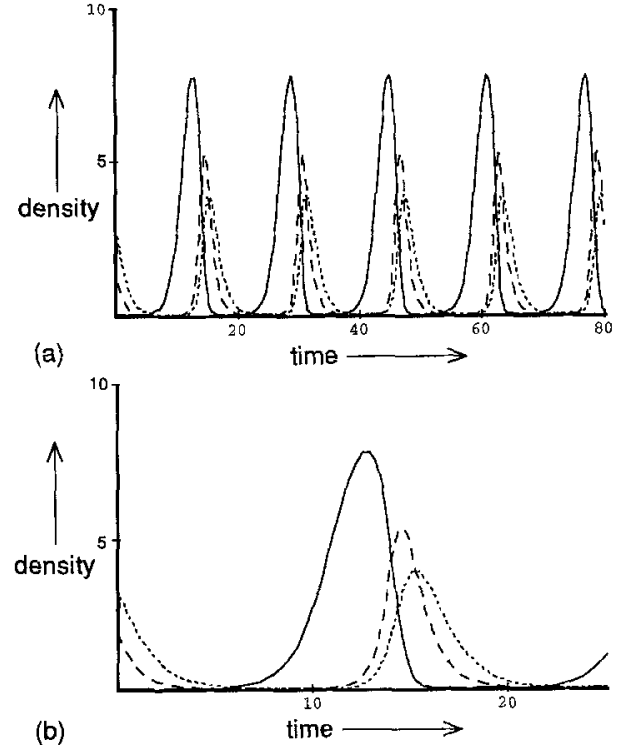

Fig. 4. (a) A typical sequence of stable oscillations of system (2) with only plants and herbivores present $(R-, N--, P-\cdots), r=1, c=10, \gamma=1, \mu=1$ and $k=1$. (b) as 4 a, blow up of one cycle. 
logistically towards carrying capacity. In the same time there is a slow build up of herbivore patches, albeit that the herbivore density is still extremely low. Because plant growth slows down as the carrying capacity is approached there comes a point where the number of dispersers gets high enough to allow for a rapid transition of almost all available free patches into herbivore patches. This is followed by the collapse of herbivore patches and the consequent production of dispersing herbivores. All these transitions are of a shorter time scale than the build up in the density of free patches. Finally the dispersers die off exponentially and the cycle starts all over again.

\section{Dynamics with all three trophic levels}

In the absence of predators the herbivores act solely as the predators of plants and thus a dispersal phase for herbivores works destabilising, as seen above. In the presence of predators the herbivores will play a double role: they remain plant predators but now are also prey to their predators. The effect of prey dispersal is stabilising. With all three trophic levels present there will be an interplay of stabilising and destabilising effects of which the outcome is not obvious.

With predators present the model allows for at most one equilibrium with plants, herbivores and predators present. The equilibrium is identical to the three-species equilibrium of the null model. This means that for low values of the carrying capacity no positive equilibrium is possible and the predators cannot maintain themselves in the system. For higher carrying capacities an equilibrium exists with plants, herbivores and predators. The equilibrium value of plants increases with increasing predator efficiency $(\eta)$, the equilibrium value of herbivores decreases and the equilibrium value of the predators increases first and then decreases again.

The stability of the three-species equilibrium is different from that of the rull model. The three-species equilibrium of the null model is always stable if it exists, whereas the equilibrium of this model can be stable or unstable. In Fig. 5 the stable and unstable domains of the three-species equilibrium are plotted for a combination of parameter values. For higher values of $\eta$, the predator efficiency in converting herbivore patches to predator patches, the equilibrium is stable. It is tempting to conclude that the stabilising action of prey dispersal is influencing the equilibrium stability but because of the stable equilibrium in the null model such a conclusion is not justified.

For lower predator efficiency instability is possible. It can be shown that $\eta<(5 / 4) \nu k / r$ is a necessary condition for instability. When herbivores are not properly controlled they dominate the dynamics and the delay, with which they react on the plant density, causes these instabilities. Apart from an influence on the destabilising delay, the predators just respond to the changes in herbivore densities. This results in a limit cycle of which the structure is the same as the one that results from plant-herbivore interaction, the difference 


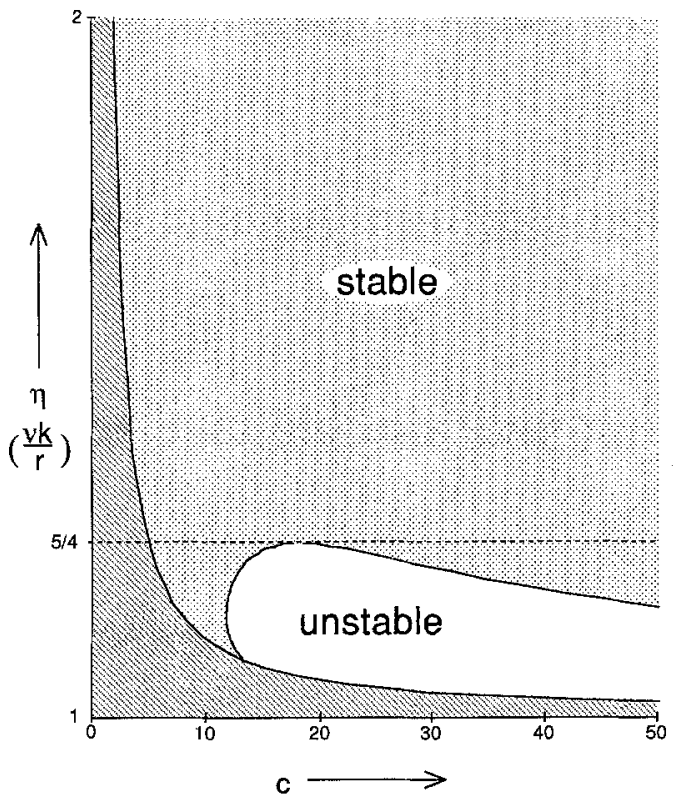

Fig. 5. Stable and unstable domains of the three-species equilibrium. In the shaded area no three-species equilibrium is possible. $r=1, \gamma=10, \mu=10, k=1$, and $\nu=1$.

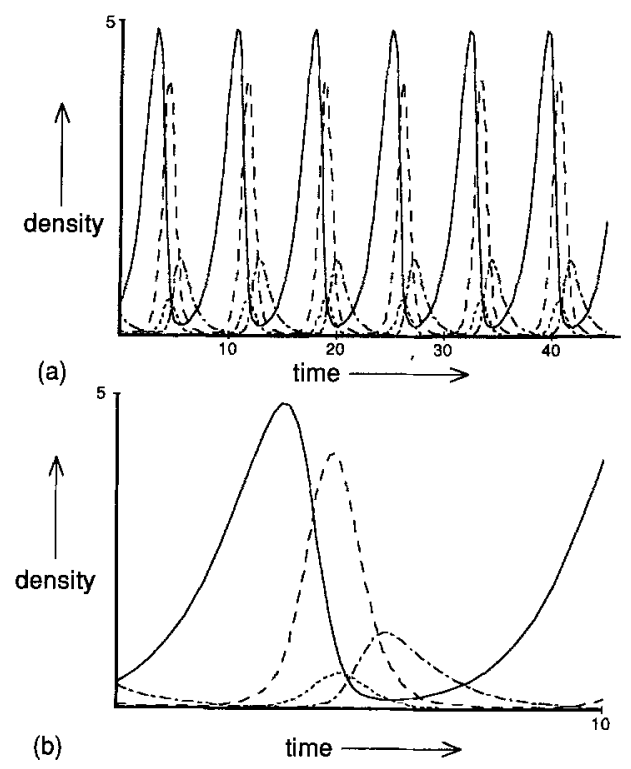

Fig. 6. (a) A sequence of stable oscillations of system (2) with plants, herbivores and predators. $(R-, N---, P-\cdots, M-\cdot-) . r=1, c=12, \gamma=7, \mu=7, k=1, \eta=1.16$ and $\nu=1$. (b) as 6a, blow up of one cycle. 
being that the herbivore peak is followed by a predator peak (Figs. $6 \mathrm{a}$ and $6 \mathrm{~b})$.

The predators cause an additional removal of herbivore patches and the presence of predators shortens average patch lifetime. Their presence has an influence on the delay in the onset of forming new patches. Whether this delay increases or decreases compared to the situation without predators, depends on the model parameters. Therefore the predators can both stabilise and destabilise the herbivore plant interaction.

\section{ON PREDATOR PERSISTENCE}

The question whether predators can invade or not was simple to answer in the section 'A simple predator-prey patch model'. Because the plant-herbivore system always settles down on its equilibrium values, it is enough to consider whether or not invasion is possible in this equilibrium. The ability of predators to invade, stability of the plant-herbivore system with respect to predators and the existence of a three-species equilibrium go strictly together. If a plant-herbivore limit cycle exists, it is not sufficient any more to study whether the predators can invade the two-species equilibrium as we did before. What should be studied now is the predator invasion over the limit cycle. This is the same as studying the stability of the limit cycle with respect to predators. In general it is difficult to find conditions for this. The techniques that are used to determine stability of equilibria, such as local stability analysis by means of Jacobian matrices and Routh Hurwitz criteria, cannot be used. Because of the simple form of this model it is possible to find criteria for invasion over the limit cycle. For this particular model invasion over the limit cycle is possible if the average herbivore patch density $(N)$ over one cycle, exceeds the herbivore patch density in the three-species equilibrium. The relation this condition has with the existence of the three-species equilibrium is that it can only be fulfilled if the three-species equilibrium exists. The reverse is not true and it is possible that the three-species equilibrium exists but that invasion over the limit cycle is not possible.

The concept of uniform persistence or permanence is closely related to the ability of predators to invade. A system is called uniformly persistent if no species will go extinct, no matter what initial conditions are chosen (Butler et al., 1986; Hofbauer and Sigmund, 1988). It is clear that a system can only be uniformly persistent if invasion is possible, because if it is not possible a small number of predators, introduced while plants and herbivores are on the limit cycle, would go extinct. In this model the ability of predators to invade over the plant-herbivore cycle and uniform persistence are equivalent properties if a single plant-herbivore limit cycle exists.

If our system is uniformly persistent nothing is said yet about the stability of the three-species equilibrium. The outcome of a predator introduction can 
be that all densities settle on their equilibrium values. It can also be that the equilibrium is unstable and that all densities keep oscillating.

If the system is not uniformly persistent introductions of predators can have several outcomes. If invasion takes place close to the plant-herbivore limit cycle (this means a small number of predators) the predators will sooner or later go extinct (Fig. 7, lower part) and plant and herbivore densities will keep oscillating as before. It is possible that extinction will occur, no matter how many predators are introduced. It is even possible that while an unstable three-species equilibrium exists, all initial conditions but one (the unstable three-species equilibrium ) will lead to predator extinction. But it is also possible that while introduction of small numbers results in extinction, introduction of larger quantities does not! If initial conditions are chosen further away from the limit cycle it is possible that all species settle down at their equilibrium density or at the three-species limit cycle, that is the predators will not go extinct (Fig. 7, upper part). With the same parameter setting different

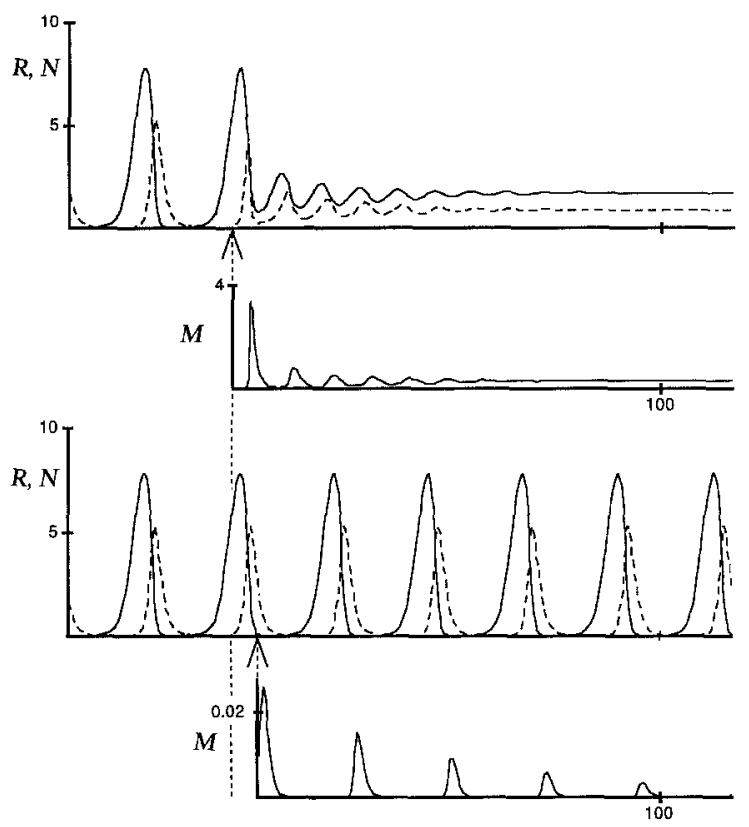

Fig. 7. The effect of introduction of an identical amount of predators $(0.01)$ in the same plantherbivore limit cycle at different moments. In the topmost graph plant (-) and herbivore $(--)$ densities are plotted against time, under it is a graph of the predator density $(-)$ after introduction, versus time. In the two bottom-most graphs the same is depicted, the only difference being the moment of introduction, introductions are indicated with arrows. In the two topmost graphs predator introduction results in a stable three-species equilibrium where in the two bottom-most graphs the predators disappear from the system, leaving the plant-herbivore cycle virtually unchanged. Note the difference in the scales for $M . r=1, c=10, \gamma=1, \mu=1, k=1$, $\eta=2.4$ and $\nu=2$. 
final states are possible. Which final state will be reached depends critically on the number of predators that are introduced and the time relative to the two-species cycle that is chosen for introduction.

The phenomenon that different initial conditions can bring the system to two different final states is called bistability. These final states of course are stable and both stable states have a domain of attraction. Disturbances can bring the system in the domain of attraction of the other stable state, resulting in a different final state. The two stable states in our system are the plantherbivore limit cycle and a three-species stable state. A sufficiently big disturbance while the three-species are in the three species stable state can result in the extinction of predators. On the other hand, if only herbivores and plants are present in a limit cycle, introduction of predators can either result in the inclusion of predators in the system, or in their rejection.

The notion of persistence is frequently used in biology and then refers to the expected time till extinction. If this expected time is relatively long compared to the time interval that has our interest the system is called persistent. Also in simulation studies the term persistence is frequently used. The normal procedure is to perform a number of simulations and to conclude that the system is persistent when the introduced predators remain present. This concept of persistence is similar to uniform persistence, but it is far from identical. If in simulations of a bistable system, like the one presented here, initial conditions are chosen repetitively outside the domain of attraction of the limit cycle one observes only predators that do not go extinct. Introductions in the same system, made under different starting conditions will result in the opposite situation and in the opposite conclusion.

\section{DISCUSSION}

A predator dispersal phase has a destabilising effect because of the delay it introduces in the response to changes in prey densities. The pattern one typically observes is that of a build up of plants, followed by an outbreak of herbivores. Outbreaks of spider mites follow qualitatively the same scenario (Burnett, 1979). The regularity in the outbreaks in the current models is caused by the unrealistically low densities that are allowed in these models (Mollison, 1991). In a more realistic situation outbreaks will occur after an occasional invasion and a more random pattern of outbreaks should be expected.

On the other hand, a prey dispersal phase has a stabilising effect because of the refuge it offers. If both mechanisms occur together, as they do in herbivore dispersal, it depends on the importance of the relations in a food web which effect overrules the other. Care should be taken when some possible stabilising or destabilising mechanism is observed in a biological system. It depends on the strength of the relations whether such a mechanism will really 
stabilise or destabilise the dynamics. Observation of a prey refuge is not a guarantee for stability.

The equivalence of the stability of the plant-herbivore equilibrium, existence of an interior equilibrium, the ability of predators to invade and uniform persistence is often true for the simplest models. With slightly more complicated systems it can easily be lost. Whenever a limit cycle can exist for two species, no matter for what reason, these equivalence relations as a rule do not hold. In such cases it is not justified to conclude from one successful (simulated) predator introduction that all introductions will be successful.

Our tri-trophic model with herbivore dispersal can be bistable; two possible stable states, namely the plant-herbivore limit cycle and a stable state with all three species present can exist. This can explain outbreaks of spider mites after pesticide treatment. It is rather unlikely that after pesticide application all predators without exception are killed, whereas spider mites are not, even if different vulnerabilities are taken into account. Yet, use of pesticides can bring the system into the domain of attraction of the plant-herbivore limit cycle. The remaining predators, not being able to bring the system back to the three-species stable state, are less and less abundant after every cycle. Finally this results in elimination of predators and continued spider mite outbreaks.

Introduction of predators in a bistable system can have two different results. It will not come as a surprise to workers in biological control that some introductions are successful whereas others are not. What we can learn from these simple models is that these failed introductions do not have to be caused by bad luck or external causes, it can be intrinsic to the biological system. What can happen is that predators at low predator density are not able to produce enough offspring during one cycle to survive the time when prey densities are low. At higher predator densities the fluctuations in the prey density are less severe, so that in the same system a predator population can be established. Introduction of natural enemies can fail because the system resists invasion, even though the same system can allow for lasting predator existence.

\section{ACKNOWLEDGEMENTS}

We thank Josef Hofbauer, Hans Metz, Karl Sigmund and especially Odo Diekmann for many helpful discussions and useful comments.

\section{REFERENCES}

Burnett, T., 1979. An acarine predator-prey population infesting roses. Res. Popul. Ecol., 29: 227-234.

Butler, G., Freedman, H.I. and Waltman, P., 1986. Uniformly persistent systems. Proc. Am. Math. Soc., 96: 425-430.

Crowley, P.H., 1978. Effective size and the persistence of ecosystems. Oecologia, 35: 185-195. 
Crowley, P.H., 1981. Dispersal and the stability of predator-prey interactions. Am. Nat., 118 : 673-701.

Diekmann, O., Metz, J.A.J. and Sabelis, M.W., 1988. Mathematical models of predator/prey/ plant interactions in a patchy environment. Exp. Appl. Acarol., 5: 319-342.

Freedman, H.I. and Waltman, P., 1977. Mathematical analysis of some three-species food-chain models. Math. Biosci., 33: 257-267.

Gard, T. and Hallam, T., 1979. Persistence in food webs I: Lotka Volterra food chains. Bull. Math. Biol., 41: 877-891.

Harrison, G.W., 1978. Global stability of food chains. Am. Nat., 114: 455-457.

Hofbauer, J. and Sigmund, K., 1988. The Theory of Evolution and Dynamical Systems: Mathematical Aspects of Evolution. Cambridge University Press, Cambridge, $341 \mathrm{pp}$.

Levin, S.A., 1976. Population dynamic models in heterogeneous environments. Annu. Rev. Ecol. Syst., 7: 287-310.

Mollison, D., 1991. Dependence of epidemic parameters and population velocity on basic parameters. Math. Biosci., 107: 255-287.

Reddingius, J. and den Boer, P.J., 1970. Simulation experiments illustrating stabilization of animal numbers by spreading of risk. Oecologia, 5: 240-284.

Sabelis, M.W. and Diekmann, O., 1988.'Overall population stability despite local extinction: the stabilizing influence of prey dispersal from predator invaded patches. Theor. Popul. Biol., 34: 169-176.

Sabelis, M.W., Diekmann, O. and Jansen V.A.A., 1991. Metapopulation persistence despite local extinction: predator prey patch models of the Lotka-Volterra type. Biol. J. Linn. Soc., 42: 267-283.

So, J.W.H., 1979. A note on global stability and bifurcation phenomenon of a Lotka-Volterra food chain. J. Theor. Biol., 80: 185-187.

Verboom, J., Lankester, K. and Metz, J.A.J., 1991. Linking local and regional dynamics in stochastic metapopulation models. Biol. J. Linn. Soc., 42: 39-55.

Zeigler, B.P., 1977. Persistence and patchiness of predator-prey systems induced by discrete event population exchange mechanisms. J. Theor. Biol., 67: 687-713. 